\title{
Through-Wall Radar Image Reconstruction Based on Time-Domain Transient Signals in the Presence of Noise
}

\author{
Anthony T. S. Ho, W. H. Tham and K. S. Low \\ School of Electrical and Electronic Engineering \\ Nanyang Technological University, \\ Nanyang Avenue, \\ Singapore 639798 \\ etsho@ntu.edu.sg
}

\begin{abstract}
Through-wall radar imaging has become a keen research topic in recent years. This is due to the emphasis on homeland security, which involves many key areas such as gathering intelligence, concealed weapons detection and domestic counter-terrorism. Other applications of through-wall radar imaging include urban warfare, hostage rescue scenarios as well as search and rescue operations.

This paper investigates the reconstruction of the position of objects behind walls based on the time-domain transient signals generated from a simulation program.

An electromagnetic wave simulation program, using the finite difference time domain (FDTD) [1] method was used to model the transient signals as they pass through walls and interact with objects of different materials. The reflected signal was then recorded by receivers at various positions behind the wall.

The difference in position between the receivers results in varying time-delays in the received signals. As a result, when the received signals are combined to form an image of the objects on the other side of the wall, the position and shape of the objects are inaccurate, resulting from the different time-delays of the radar returns. Migration techniques such as normal move-out (NMO) are used to compensate and reconstruct a more accurate shape of the objects.

Time-delay correction applied to the signals is dependent on the ability to detect the presence of the transmitted pulse. This problem becomes increasingly difficult in the presence of noise. Pre-processing techniques such as low-pass filtering and Wiener filtering were applied to improve the signal detection.
\end{abstract}

Keywords - Finite-Difference Time-Domain (FDTD), Normal Move-out (NMO), Through-Wall Radar Imaging.

\section{INTRODUCTION}

There are many occasions when it is desirable to inspect the interior of a room or building before physically entering it. This is especially so when entering the room might pose a serious danger to the person involved. These situations include civil engineering, search and rescue and law enforcement scenarios.

Through-wall radar imaging is the technology that addresses this problem and has become a keen research topic in recent years. Its importance is due to the increased emphasis on homeland security, which involves many key areas such as gathering intelligence, concealed weapons detection and domestic counter-terrorism. Other applications of through-wall radar imaging include urban warfare, hostage rescuing scenarios as well as search and rescue operations.

Furthermore, the study and use of ultra-wideband (UWB) technology has also gained momentum in the last few years due to the approval of its use commercially by the Federal Communications Commission (FCC) in 2002. The large available bandwidth for UWB and its performance in dispersive environments make it ideal for indoor use in highclutter environments. Coupled with the fact that UWB provides high spatial resolution, the use of UWB in throughwall radar imaging can be seen as an obvious choice.

With the use of multiple receivers and transmitters at different positions, and correcting for the time-delay in the received signals using normal moveout (NMO), an image of the objects within the room can be reconstructed. However, the accuracy of the positions of the objects may vary due to the presence of noise.

A computer program based on the finite difference time domain (FDTD) method was used to simulate the room environment as well as the transmitted UWB pulse. Multiple receivers were placed behind the wall to record the reflected signals from the objects in the room. The transmitter was then moved to various positions to obtain different sets of data. The image of the room was reconstructed by applying NMO on the various sets of received data.

Section II will introduce the use of FDTD to simulate the UWB pulse as well as the room environment. Section III highlights the use of NMO on the received signal data. Section IV will show the results obtained from the application of NMO on the received signals generated using the FDTD simulations. 


\section{FINITE DIFFERENCE TIME DOMAIN (FDTD)}

\section{A. Maxwell's Equations}

Maxwell's equations for linear, isotropic, non-dispersive materials are

$$
\begin{gathered}
\frac{\partial \mathbf{B}}{\partial t}=-\nabla \times \mathbf{E} \\
\frac{\partial \mathbf{D}}{\partial t}=\nabla \times \mathbf{H}-\mathbf{J}
\end{gathered}
$$

Taking only two dimensions (i.e. electric and magnetic fields do not vary in the z-direction) and considering only the transverse-electric mode $\left(\mathrm{TE}_{\mathrm{z}}\right)$, Maxwell's equations can be expanded into

$$
\begin{gathered}
\frac{\partial H_{z}}{\partial t}=\frac{1}{\mu}\left[\frac{\partial E_{x}}{\partial y}-\frac{\partial E_{y}}{\partial x}\right] \\
\frac{\partial E_{x}}{\partial t}=\frac{1}{\varepsilon}\left[\frac{\partial H_{z}}{\partial y}-\sigma E_{x}\right] \\
\frac{\partial E_{y}}{\partial t}=\frac{1}{\varepsilon}\left[-\frac{\partial H_{z}}{\partial x}-\sigma E_{y}\right]
\end{gathered}
$$

\section{B. Finite Difference Formulation}

Equations (3), (4) and (5) can be expressed in terms of finite differences. Based on the notation and formulation in [2], we then have the following update equations for the electric and magnetic fields

$$
\begin{gathered}
\left.H_{z}\right|_{i+1 / 2, j+1 / 2} ^{n+1 / 2}=\left.H_{z}\right|_{i+1 / 2, j+1 / 2} ^{n-1 / 2}+ \\
\frac{\Delta t}{\mu}\left[\frac{\left.E_{x}\right|_{i+1 / 2, j+1} ^{n}-\left.E_{x}\right|_{i+1 / 2, j} ^{n}}{\Delta y}-\right. \\
\left.\frac{\left.E_{y}\right|_{i+1, j+1 / 2} ^{n}-\left.E_{y}\right|_{i, j+1 / 2} ^{n}}{\Delta x}\right] \\
\left.E_{x}\right|_{i+1 / 2, j} ^{n+1}=\left.\left(\frac{2 \varepsilon-\sigma \Delta t}{2 \varepsilon+\sigma \Delta t}\right) E_{x}\right|_{i+1 / 2, j} ^{n}+ \\
\frac{2 \Delta t}{2 \varepsilon+\sigma \Delta t}\left[\frac{\left.H_{z}\right|_{i+1 / 2, j+1 / 2} ^{n+1 / 2}-\left.H_{z}\right|_{i+1 / 2, j-1 / 2} ^{n+1 / 2}}{\Delta y}\right]
\end{gathered}
$$

$$
\begin{aligned}
\left.E_{y}\right|_{i, j+1 / 2} ^{n+1}= & \left.\left(\frac{2 \varepsilon-\sigma \Delta t}{2 \varepsilon+\sigma \Delta t}\right) E_{y}\right|_{i, j+1 / 2} ^{n}- \\
& \frac{2 \Delta t}{2 \varepsilon+\sigma \Delta t}\left[\frac{\left.H_{z}\right|_{i+1 / 2, j+1 / 2} ^{n+1 / 2}-\left.H_{z}\right|_{i-1 / 2, j+1 / 2} ^{n+1 / 2}}{\Delta x}\right]
\end{aligned}
$$

\section{Berenger's Perfectly Matched Layer (PML)}

A problem with using the FDTD method is that as the scattered electromagnetic fields reach the boundary of the simulation domain, they are reflected back, which results in undesirable interference. As a result, absorbing boundary conditions need to be applied at the boundary of the simulation domain to accurately simulate the electromagnetic waves propagating out into free space.

Absorbing boundary conditions developed by Berenger [3, 4] and Mur [5] have been used in many FDTD models to solve for the boundary problem. Due to its superior absorption of the electromagnetic waves at any frequency and incidence angle, Berenger's perfectly matched layer (PML) was chosen for the current simulation model.

\section{NORMAL MOVEOUT (NMO)}

\section{A. Introduction}

NMO [6] is the difference in time-delay between the transmitted signal and received signals from multiple receivers which are at varying distances away from the transmitter (Fig. $1)$.

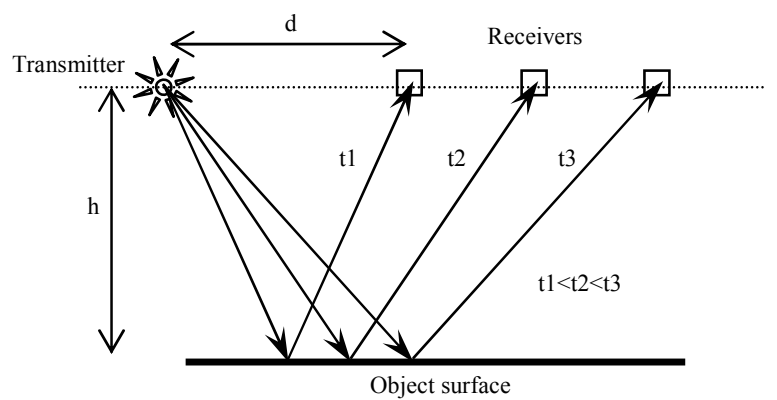

Figure 1 Time-delay due to varying distance of different receivers from the transmitter

\section{B. NMO Correction}

To correct for the time-delay caused by NMO, the received signal at each individual receiver must be time-shifted appropriately according to

$$
T_{i}=\frac{2 \sqrt{h_{i}^{2}+\left(d_{i} / 2\right)^{2}}}{c}
$$

where $h_{i}$ is the distance from the receiver to the object surface, $d_{i}$ is the distance from the transmitter to the receiver and $c$ is the speed in which the transmitted pulse is traveling at. 


\section{Stacking}

A single transmitter position will result in only a single set of received signal data. However, this is not sufficient to obtain the required position and shape of the objects within the room. To do so, the transmitter must be moved to different positions, and the different sets of received signals must be added or "stacked" together. This eliminates the unwanted spurious reflections and also helps improve the signal-to-noise (SNR) ratio.

\section{IV.RESULTS}

\section{A. Setup Parameters}

The set up parameters for the FDTD simulation are shown in Fig. 2. The simulation domain consists of a 800-by-450 cell FDTD grid. A gypsum wall $\left(\varepsilon_{\mathrm{r}}=2.8, \sigma=0.15\right)$ and metal object $\left(\varepsilon_{\mathrm{r}}=1.0, \sigma=1 \times 10^{7}\right)$ were used for this simulation. For display purposes, the receivers have been placed at every cell position (i.e. there are 800 receivers along the face of the wall). The transmitter is moved along the edge of the wall at cell positions $325,350,375,400,425,450$ and 475 .

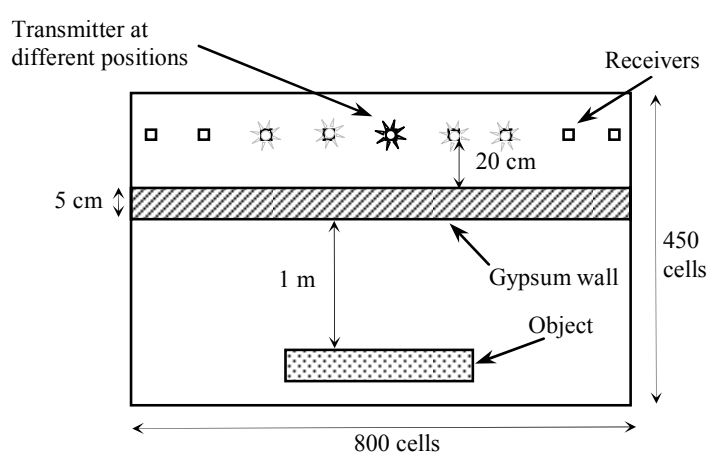

Figure 2 Layout of simulation domain

\section{B. UWB Input Pulse}

The transmitted UWB pulse is given by

$$
x(t)=\cos \left(2 \pi f_{c} t\right) \exp \left(-\frac{t^{2}}{u^{2}}\right)
$$

where $f_{c}$ is the center frequency and $u$ determines the bandwidth of the pulse. For our simulation, a pulse with center frequency $4 \mathrm{GHz}$ and bandwidth of $2 \mathrm{GHz}$ was used. Fig. 2 shows the pulse shape of the transmitted pulse and its corresponding Fourier spectrum.
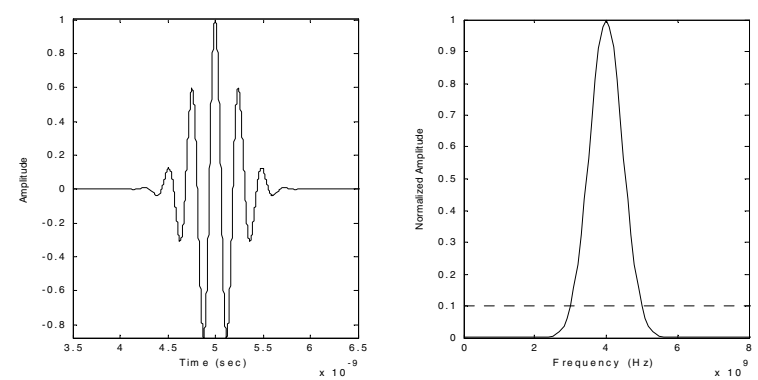

Figure 3 Transmitted UWB pulse and its spectrum

\section{Received Signal}

The receivers record the received signal at every time-step from the start of the simulation. Fig. 4 shows the received signal after 2000 time-steps for the transmitter at position 400 . Note that the interaction of the reflected wave from the wall has been time-gated out. Thus, the signal in Fig. 4 purely represents the reflection from the metal object.

The approximate position of the object can be seen from the strong signal at the center. However, the exact width of the object cannot be determined. Furthermore, the parabolic shape of the received signal (due to the time-delay between the transmitted pulse and the receivers at different positions) can be seen clearly.

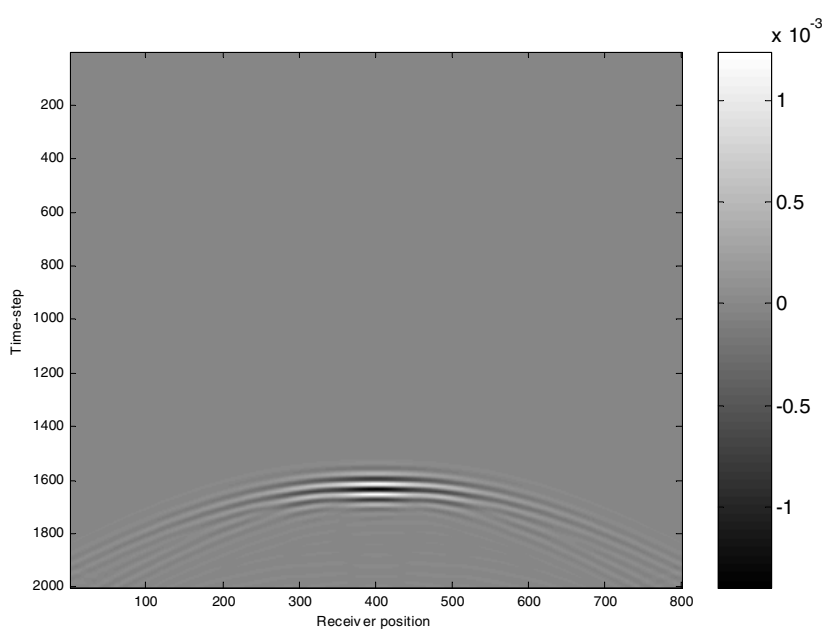

Figure 4 Received signal with transmitter placed at the center of the simulation domain (i.e. cell position 400)

\section{NMO Correction}

NMO correction was then performed on the received signal to adjust for the time-delays. Fig. 5 shows the corrected received signal. As can be seen, the received signal now resembles the shape of the original object (which was flat).

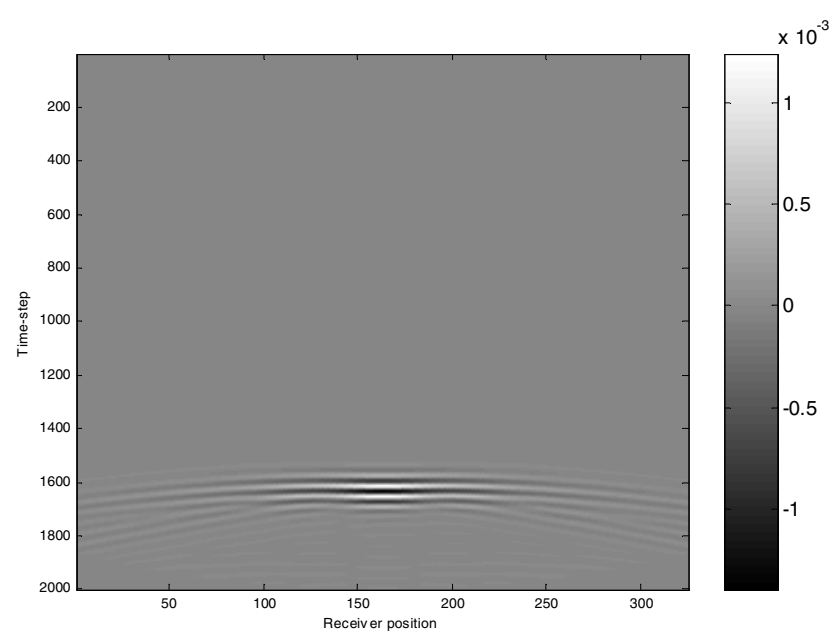

Figure 5 Received signal with NMO correction 


\section{E. Stacking}

The transmitter was then moved to a different position and a different set of received signal data was obtained. All the received signals for the different transmitter positions were then "stacked".

Fig. 6 shows the results after stacking. Some of the spurious reflections have been eliminated, resulting in a "cleaner" signal. Also, notice the improved signal-to-noise ratio from the increase in signal amplitude. Also, the actual width of the object (shown as dotted lines in Fig. 6) shows good correlation with the received signal.

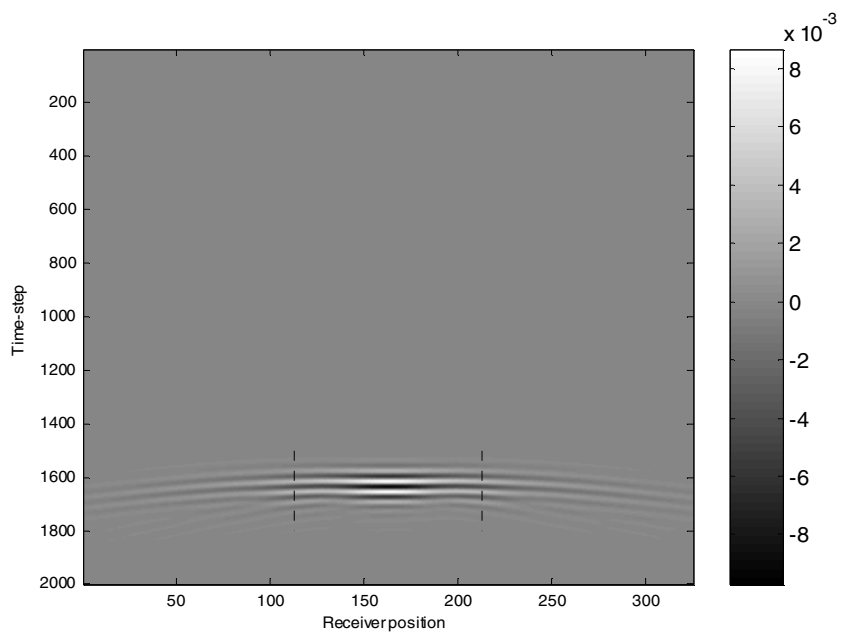

Figure 6 Improved signal after stacking

\section{F. Addition of Noise}

The received data generated thus far have been ideal, in that no noise was present. To simulate an actual environment, white Gaussian noise was added to the received signal data (Fig. 7). The signal-to-noise ratio (SNR) was approximately $20 \mathrm{~dB}$.

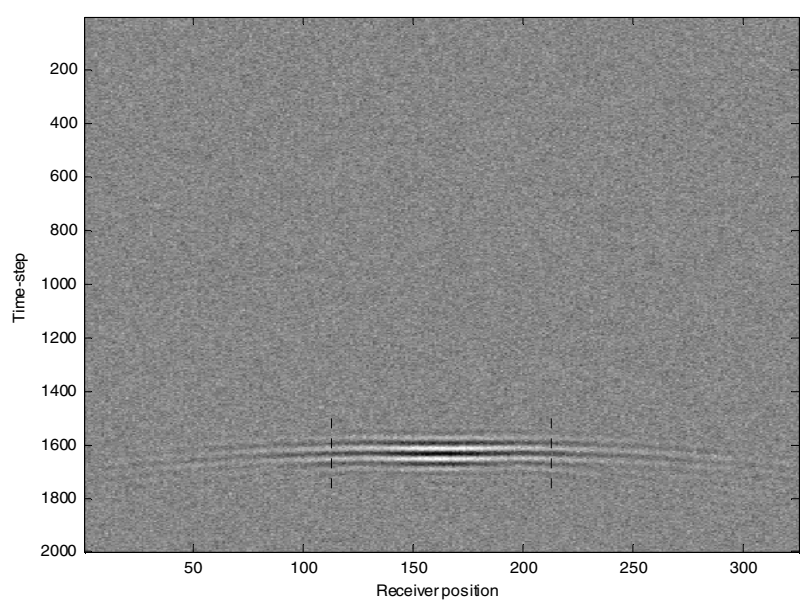

Figure 7 Received signal with noise added $(\mathrm{SNR}=20 \mathrm{~dB})$
A median filter was then applied to improve on the SNR. Fig. 8 shows the received signal data after filtering, with an improved SNR of approximately $33 \mathrm{~dB}$.

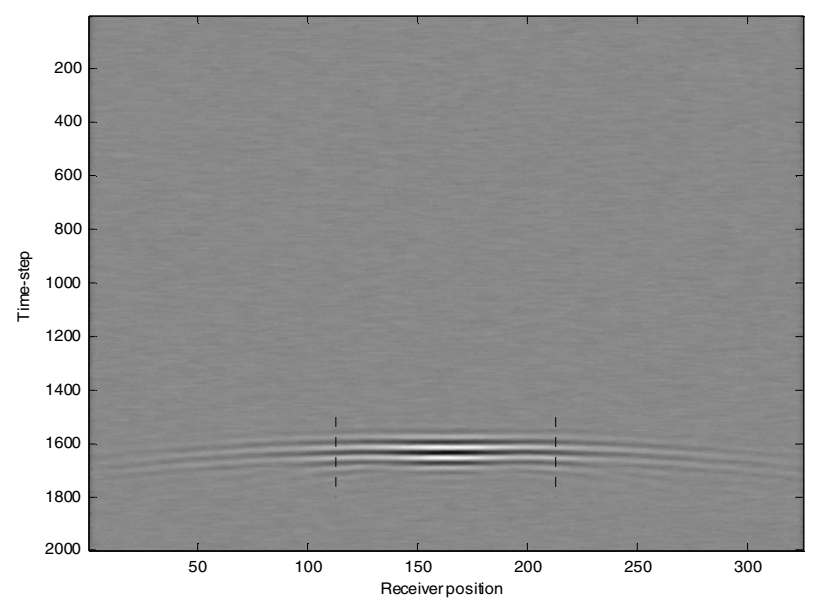

Figure 8 Received signal after filtering $(\mathrm{SNR}=33 \mathrm{~dB})$

\section{CONCLUSION}

A simulation has been designed to model the transmission of an UWB pulse for the purpose of through-wall imaging.

Normal moveout (NMO) was used to correct for the timedelay due to varying transmitter to receiver distances. The various receiver signals from different transmitter positions were then stacked to obtain an improvement in the signal-tonoise ratio.

To model a real environment, noise was added. Filtering was then applied to improve on the SNR (from $20 \mathrm{~dB}$ to 33 $\mathrm{dB}$ ). Future work will involve adding and comparing other objects of varying shapes, sizes as well as different materials under noisy conditions.

\section{REFERENCES}

[1] [1] K. S. Yee, "Numerical solutions of initial boundary value problems involving Maxwell's equations in isotropic media," IEEE Trans. Antennas Propagat., vol. AP-14, pp. 310-317, May 1966.

[2] A. Taflove and Susan C. Hagness, Computational Electrodynamics: The Finite-Difference Time-Domain Method, $2^{\text {nd }}$ Edition, Artech House, 2000.

[3] J. P. Berenger, "A perfectly matched layer for the absorption of electromagnetic waves," Journal of Computational Physics, vol. 114, pp. 185-200, 1994

[4] J. P. Berenger, "Perfectly matched layer for the FDTD solution of wavestructure interaction problems," IEEE Trans. Antennas Propagat., vol. 44, pp. 110-117, Jan 1996.

[5] G. Mur, "Absorbing boundary conditions for finite-difference approximation of the time-domain electromagnetic-field equations," IEEE Trans. Electromagn. Compat., vol. 23, pp. 1073-1077, 1981.

[6] J. F. Boyce, "Seismic Processing and Image Analysis", J. Phys. D: Appl. Phys. 19, pp. 397-415, 1986. 